\title{
The Effects of GLUT1 on the Survival of Head and Neck Squamous Cell Carcinoma
}

\author{
Shengjiao Lia, Xiaoning Yang ${ }^{c}$ Peng Wang ${ }^{a, b}$ Xing Ran ${ }^{a, b}$ \\ aDepartment of Oral and Maxillofacial Surgery, Stomatology Hospital, Tongji University, Shanghai; \\ bLaboratory of Oral Biomedical Science and Translational Medicine, School of Stomatology, Tongji \\ University, Shanghai; 'Department of Obstetrics and Gynecology, Shanghai Pudong Hospital, Shanghai
}

\section{Key Words}

Glucose transporter-1 - Head and neck squamous cell carcinoma • Nuclear factor-kappa B

\begin{abstract}
Background/Aims: Cancer cells require increased nutrient uptake to support a high rate of proliferation, and the overexpression of glucose transporters, in particular GLUT1, is a common characteristic of human malignancies. Here, we investigated the relationship between the expression of GLUT1 and cell viability, colony forming ability and apoptosis of head and neck squamous cell carcinoma (HNSCC) in vitro and in a xenograft mouse model in vivo. Methods: Lentiviral mediated overexpression and knock-down of GLUT1 was performed in two oral cancer cell lines (CAL27 and SCC25). QRT-PCR and Western blot analysis were used to detect the mRNA and protein expression of GLUT1 and nuclear factor-kappa B (NFKB) p65 subunit. Cell viability and apoptosis were assessed by MTT and flow cytometry analyses, respectively. Colony formation assays were performed by staining with $0.5 \%$ crystal violet. The role of GLUT1 in HNSCC was examined in vivo through the generation of a CAL27 (or CAL27 with different transfections) nude mice xenograft model of HNSCC. Results: GLUT1 overexpression promoted cell viability and colony formation whereas GLUT1 silencing had the opposite effect. GLUT1 knock-down significantly increased the number of Annexin V positive cells in both cell lines and GLUT1 overexpression had the opposite effect, indicating that GLUT1 modulates apoptosis. Xenograft mouse models of GLUT1 knockdown and overexpression showed that GLUT1 expression was associated with poor survival and increased tumor growth. GLUT1 overexpression significantly upregulated the expression of NFKB-p65, and this effect was reversed by inhibition of GLUT1 expression. Conclusions: GLUT1 expression plays an important role in the survival of HNSCC, and its effects may be associated with the activation of the NFkB pathway.
\end{abstract}


Li/Yang/Wang/Ran: Relationship between GLUT1 and HNSCC

\section{Introduction}

The transport of glucose across the cell membrane is mediated by two types of transporters, the sodium-dependent glucose transporter family (SGLT) and the GLUT family of transporters [1]. Facilitative glucose transporters (GLUTs) mediate the energy independent transport of glucose along a concentration gradient [2]. In mammals, 14 GLUTs have been identified and each possesses a different affinity for glucose and other hexoses. The most widely expressed transporter is GLUT1, and it is often upregulated during oncogenesis in different tissues $[3,4]$. Tumor cells show an increased rate of aerobic glycolysis known as the Warburg effect that is supported by an increase in glucose transport and consumption $[5,6]$. Increased expression of GLUT1 has been detected in several malignancies including breast cancer [7], thyroid cancer [8], head and neck cancer [9], and lung cancer [10] and its overexpression has been correlated with poor prognosis in several cancers including head and neck cancer $[11,12]$.

Head and neck squamous cell carcinoma (HNSCC) includes malignancies arising from the mucosal lining of the oral cavity, oropharynx, nose, hypopharynx and larynx [13]. According to the American Cancer Society, an estimated 52,610 new cases of head and neck cancer will be diagnosed in 2012, and approximately 11,500 will result in death [14]. Approximately $60 \%$ of patients have locally or regionally advanced disease at diagnosis, and prognosis remains poor with a 5-year survival rate of $56 \%$ among patients with oral cavity or pharyngeal cancer and $41 \%$ for those with laryngeal cancer. Advanced disease is often treated with multimodal therapy consisting of surgery, radiotherapy and/or chemotherapy [15]. Cisplatin is commonly used as a single agent or in combination in chemoradiotherapy (CRT) regimens. However, the effectiveness of platinum agents is often limited by their toxicity and the development of resistance.

Activation of NFKB is associated with several human malignancies, which is partly attributed to its ability to activate the transcription of genes that promote cell survival, thus contributing to tumor growth and resistance to chemotherapy [16]. We found that inhibition of GLUT1 expression can sensitize CAL27 cells to cisplatin in our previous study [17]. The present study aimed to investigate the relationship between oral cancer cell proliferation, apoptosis, tumor growth and the expression of glucose transporters and to elucidate the mechanisms underlying the effect of increased expression of GLUT1 on tumorigenesis in HNSCC.

\section{Materials and Methods}

Cell lines and culture

The HNSCC cell lines CAL27 and SCC25 were obtained from the American type culture collection. SCC25 cells were grown in Dulbecco's minimal essential medium (DMEM)/Ham's F-12 medium supplemented with $20 \%$ fetal bovine serum (FBS, HyClone, Logan, UT, USA), hydrocortisone ( $40 \mathrm{ng} / \mathrm{mL}$ ) and sodium pyruvate (1 mM). CAL27 cells were grown in DMEM supplemented with 10\% FBS.

\section{Lentivirus vector structure and transfection}

Lenti-shGLUT1 was constructed by inserting the oligo DNA 5'-CTT CTA TTA CTC CAC GAG CAT-3' into the PLVTHM lentiviral vector (System Biosciences, SBI, Mountain View, CA) at the MluI/ClaI site. Lentiviral vectors were produced by transient transfection of $293 \mathrm{~T}$ cells as described previously [18]. To generate constructs expressing GLUT1, sequences amplified by PCR [gi|166795298|ref|NM_006516.2| Homo sapiens solute carrier family 2 (facilitated glucose transporter), member 1 (SLC2A1)] were cloned into the lentiviral vector pCDH-CMV (System Biosciences). Restriction Enzyme cutting sites were as follows:

EcoRI and NotI, product length $2850 \mathrm{bp}$ :

GLUT1- EcoRI: 5'-GGAATTCAGGCAAGAGGTAGCAACAGC-3';

GLUT1-NotI: 5'-TGCGGCCGCCACGGCTGGCACAAAACTA-3'. 
Li/Yang/Wang/Ran: Relationship between GLUT1 and HNSCC

High titer lentiviruses were generated by transfection of 293T cells with pCDH-CMV-GLUT1, and CAL27 and SCC25 cell lines were incubated with infectious lentivirus medium of Lenti-shGLUT1 or pCDHCMV-GLUT1.

\section{Cell viability assays}

Cell viability was assessed using the MTT assay as described previously with minor modifications $[19,20]$. Briefly, cells with different transfection were seeded in 96 -well plates at a density of $5 \times 10^{3}$ and grown for $24 \mathrm{~h}$. Cells were then treated with different concentrations of cisplatin for $72 \mathrm{~h}$ as indicated. MTT (3-[4,5-dimethylthiazol-2-yl]-2,5 diphenyltetrazolium bromide, Sigma) was added to each well to a final concentration of $0.5 \mathrm{mg} / \mathrm{mL}$ and incubated for $4 \mathrm{~h}$ in a moist chamber at $37^{\circ} \mathrm{C}$. The medium was then removed and $0.2 \mathrm{~mL}$ DMSO was added. The absorbance (A) of the formazan product was measured at 570 $\mathrm{nm}$ by a microtiter plate reader (Bio-Rad Labs, Sunnyvale, CA). $\mathrm{IC}_{50}$ (the concentration required for $50 \%$ inhibition of cell growth) values were calculated by interpolation from a sigmoidal dose response curve fit of the log transformed survival data, derived using GraphPad Prism version 4.0 for Mac OS X (GraphPad Software Inc., San Diego, USA). The cell viability rate was calculated by the following formula: Cell viability rate $(\%)=\left(A_{570 \text { treated }} / A_{570 \text { control }}\right) \times 100 \%$.

\section{Colony formation assay}

Cells with different transfections were trypsinized and seeded for colony formation assay in six-well plates at a density of 200 cells per well. Cells were cultured for 7 days, and then stained with $0.5 \%$ crystal violet in methanol for $15 \mathrm{~min}$. Stained colonies larger than $1 \mathrm{~mm}$ in diameter were counted. Each colony formation assay was carried out in triplicate and repeated at least two times.

\section{Apoptosis assay}

Apoptosis was assayed using an Annexin V-FITC/PI Apoptosis Detection Kit (BD Pharmingen, CA, USA) according to the manufacturer's instructions. Cells were cultured and treated as indicated. Control and transfected cells were harvested by trypsinization, resuspended by adding $500 \mu \mathrm{l}$ binding buffer ( $\mathrm{pH}$ 7.5, $10 \mathrm{mM}$ HEPES, $2.5 \mathrm{mM} \mathrm{CaCl}_{2}$ and $140 \mathrm{mM} \mathrm{NaCl}$ ) and stained with AnnexinV-FITC for $30 \mathrm{~min}$ at room temperature. After washing and resuspension in PI binding buffer, cells were detected by flow cytometry (FACScan, Becton Dickinson, USA), and analyzed using Cell Quest Software (BD Biosciences, Franklin Lakes, NJ, USA).

\section{Quantitative real time PCR}

Quantitative real-time PCR (qRT-PCR) analysis was performed using total RNAs extracted from cells with the RNeasy mini kit (Qiagen, Inc., CA, USA). RNA was reverse transcribed with the RetroScript kit (Ambion, Austin, TX) according to the manufacturer's instructions. qRT-PCR was performed using SYBR green Rox Master Mix (Qiagen).

Primer sequences used in qRT-PCR were are follows:

GLUT1: forward, 5'-TTG GCT CCG GTA TCG TCA AC-3', reverse, 5'-GCC AGG ACC CAC TTC AAA GA-3'; NFkB-p65 subunit: forward, 5'-TGG ACG ATC TGT TTC CCC TCA-3', reverse, 5'-ATT GGG TGC GTC TTA GTG GT-3'; GAPDH: forward, 5'- CTA CCC CCA ATG TGT CCG TC-3', reverse, 5'- TGA AGT CGC AGG AGA CAA CC-3'.

\section{Western blotting}

Expression of proteins was detected by Western blot assay as described previously [21]. Briefly, cell extracts and tumor lysates were separated by SDS-PAGE followed by transfer to nitrocellulose membranes. Membranes were blocked with 5\% milk in TBS-T (TBS containing Tween 20) for $30 \mathrm{~min}$ to $1 \mathrm{~h}$ and incubated in primary antibody (Santa Cruz, CA, USA) overnight at $4^{\circ} \mathrm{C}$ followed by incubation in peroxidase-conjugated goat anti-rabbit IgG (1:500, Santa Cruz, CA, USA) for 1 to $2 \mathrm{~h}$. Proteins were visualized by autoradiography and films were scanned and quantified using a gel image analysis system (Bio-Rad, Hercules, USA). Primary antibodies and dilutions used were anti-GLUT1 (1:2000), anti-NFKB-p65 (1:500) and anti-GAPDH (1:1000).

\section{HNSCC xenograft tumor model}

All animal experiments were approved by the Animal Research Committee atShanghai Tongji University and were carried out in accordance with established International Guiding Principles for Animal Research. 
Li/Yang/Wang/Ran: Relationship between GLUT1 and HNSCC

Five week-old female athymic nude mice (15 per group), used for in vivo experiments, were obtained from Shanghai Laboratory Animal Center (SLAC, Shanghai, China). CAL27 cells or lentivirus transfected cells (4 $\times 10^{6}$ cells per mouse) were injected subcutaneously into the flanks of mice to generate xenograft tumors [22]. Animals were maintained in a sterile environment and given free access to sterile water and food. One week after implantation, an investigator unaware of the treatment group measured the longest and shortest tumor diameters with electronic digital calipers every three days for 21 days. Tumor volume (TV) was calculated according to the following formula: TV $\left(\mathrm{mm}^{3}\right)=$ length $\times$ width $^{2} \times 0.5$. After 28 days, all mice were humanely sacrificed, and tumor xenografts were harvested and snap-frozen.

\section{Statistical analysis}

All data were expressed as means \pm SD from triplicate experiments performed in a parallel manner unless otherwise indicated. For statistical analysis of the data, group means were compared by one-way ANOVA, and Bonferroni's test was used to identify differences between groups. Statistical differences were considered significant at the ${ }^{*} \mathrm{P}<0.05$ or ${ }^{* *} \mathrm{P}<0.01$ level. All the figures shown in this article were obtained from at least three independent experiments.

\section{Results}

GLUT1 expression is correlated with cisplatin resistance

To examine the role of GLUT1 in squamous cell carcinoma, the GLUT1 gene was overexpressed or knocked-down by infection of CAL27 and SCC25 cells with lentiviral vectors carrying GLUT1 (GLUT1) or shRNA targeting GLUT1 (shGLUT1). The parental lines served as normal control (NC). GLUT1 mRNA expression assessed by QRT-PCR and normalized to the expression of GAPDH was significantly higher in Lenti-GLUT1 transfected cells, than in the NC $(\mathrm{P}<0.01)$, whereas it was significantly lower in Lenti-shGLUT1 transfected cells $(\mathrm{P}<0.01$, Fig. 1A). Western blot analysis and quantification of band density relative to the expression of GAPDH showed that GLUT1 protein levels followed the same trend as GLUT1 mRNA levels (Fig. 1B).

To examine the association between GLUT1 expression and cisplatin resistance in squamous cell carcinoma, two oral cancer cell lines (CAL27 and SCC25) transfected with lentivirus as indicated were incubated in increasing doses of cis-diamminedichloroplatinum (CDDP, cisplatin). Assessment of cell viability using the MTT assay after exposure of cells to increasing concentrations of CDDP for $72 \mathrm{~h}$ showed that cells overexpressing GLUT1 were more resistant to cisplatin than the NC, whereas shGLUT1 cells were sensitive to CDDP (Fig. $1 \mathrm{C}$ and $\mathrm{D})$. The $\mathrm{IC}_{50}$ values for CDDP were higher in GLUT1 overexpressing cells than in NC and shGLUT1 cells (Table 1). These results indicated that GLUT1 expression is correlated with cisplatin resistance in HNSCC cells.

\section{GLUT1 expression modulates cell apoptosis in oral cancer cell lines}

To determine whether the effect of modulation of GLUT1 expression was mediated by apoptosis, CAL27 and SCC25 cells expressing Lenti-shGLUT1 or Lenti-GLUT1 were stained with fluorescein-isothiocyanate (FITC)-conjugated Annexin V and propidium iodide (PI) and analyzed by flow cytometry to determine the percentage of Annexin $\mathrm{V}$ positive apoptotic cells (Fig. 2A and 2B). The results showed that GLUT1 knock-down significantly induced apoptosis, increasing the percentage of Annexin $\mathrm{V}$ positive cells from $2.3 \%$ and $5.6 \%$ in untreated CAL27 and SCC25 cells to $52.3 \%$ and $39.4 \%$, respectively $(\mathrm{P}<0.01)$. These result indicated that the effect of GLUT1 knock-down was mediated by the induction of apoptosis in HNSCC cells.

\section{GLUT1 expression modulates colony formation ability in oral cancer cell lines}

To determine whether GLUT1 knock-down or overexpression affected colony formation, CAL27 and SCC25 cells transfected with different lentiviral constructs were cultured in 10 $\mathrm{cm}$ tissue culture dishes, stained with crystal violet and stained colonies were counted (Fig. 


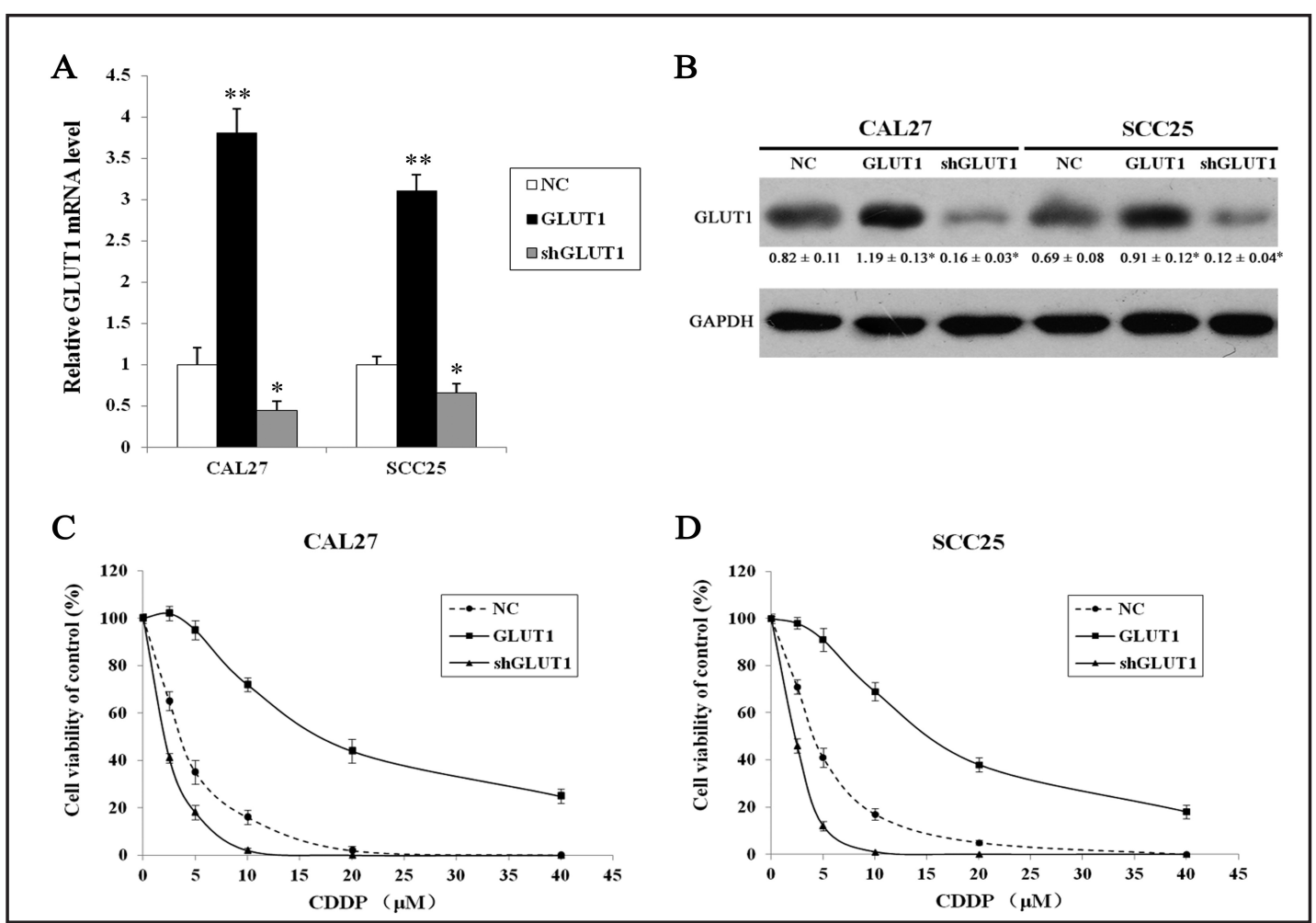

Fig. 1. GLUT1 expression is correlated with cisplatin resistance in oral cancer cells. The GLUT1 gene was overexpressed or knocked-down by infection of CAL27 and SCC25 cells with lentiviral vectors carrying GLUT1 (GLUT1) or shRNA targeting GLUT1 (shGLUT1). The parental lines served as normal control (NC). (A) GLUT1 mRNA was detected by Q RT-PCR, normalized to GAPDH expression and depicted as fold-change relative to the NC group. (B) GLUT1 protein expression was detected by Western blotting. GAPDH was used as the loading control. Quantification of GLUT1 protein level was performed by densitometry and normalized to the level of GAPDH. (C-D) CAL27 (C) and SCC25 (D) cells transfected with lentivirus as indicated were incubated in increasing doses of cisplatin (CDDP) for $72 \mathrm{~h}$ and cell viability was assessed by the MTT assay. All values are the means $\pm \mathrm{SD}$ of three replicates, ${ }^{*} \mathrm{P}<0.05,{ }^{* *} \mathrm{P}<0.01 \mathrm{VS}$ NC of each cell line.

Table 1. $\mathrm{IC}_{50}(\mu \mathrm{M})$ values of CAL27 and SCC25

\begin{tabular}{|c|c|c|c|c|c|c|}
\hline & \multicolumn{3}{|c|}{ CAL27 } & \multicolumn{3}{|c|}{ SCC25 } \\
\hline & $\mathrm{NC}$ & GLUT1 & shGLUT1 & $\mathrm{NC}$ & GLUT1 & shGLUT1 \\
\hline $\mathrm{IC}_{50}$ & $3.857 \pm 0.091$ & $16.157 \pm 1.471$ & $1.621 \pm 0.054$ & $4.522 \pm 0.149$ & $15.483 \pm 1.027$ & $1.695 \pm 0.068$ \\
\hline
\end{tabular}

2C and 2D). Consistent with the cell viability results, GLUT1 silencing significantly decreased the number of colonies formed in both cell lines $(\mathrm{P}<0.01)$ whereas GLUT1 overexpression significantly increased the number of colonies $(\mathrm{P}<0.05)$.

GLUT1 expression modulates the NFKB pathway

To further examine the association between NFKB signaling and the effects of GLUT1 on HNSCC cells growth, the expression of the NFKB-p65 subunit was determined at the mRNA and protein levels in CAL27 and SCC25 cells in correlation with GLUT1 expression. The results of QRT-PCR showed that the mRNA levels of GLUT1 and NFKB-p65 were significantly 


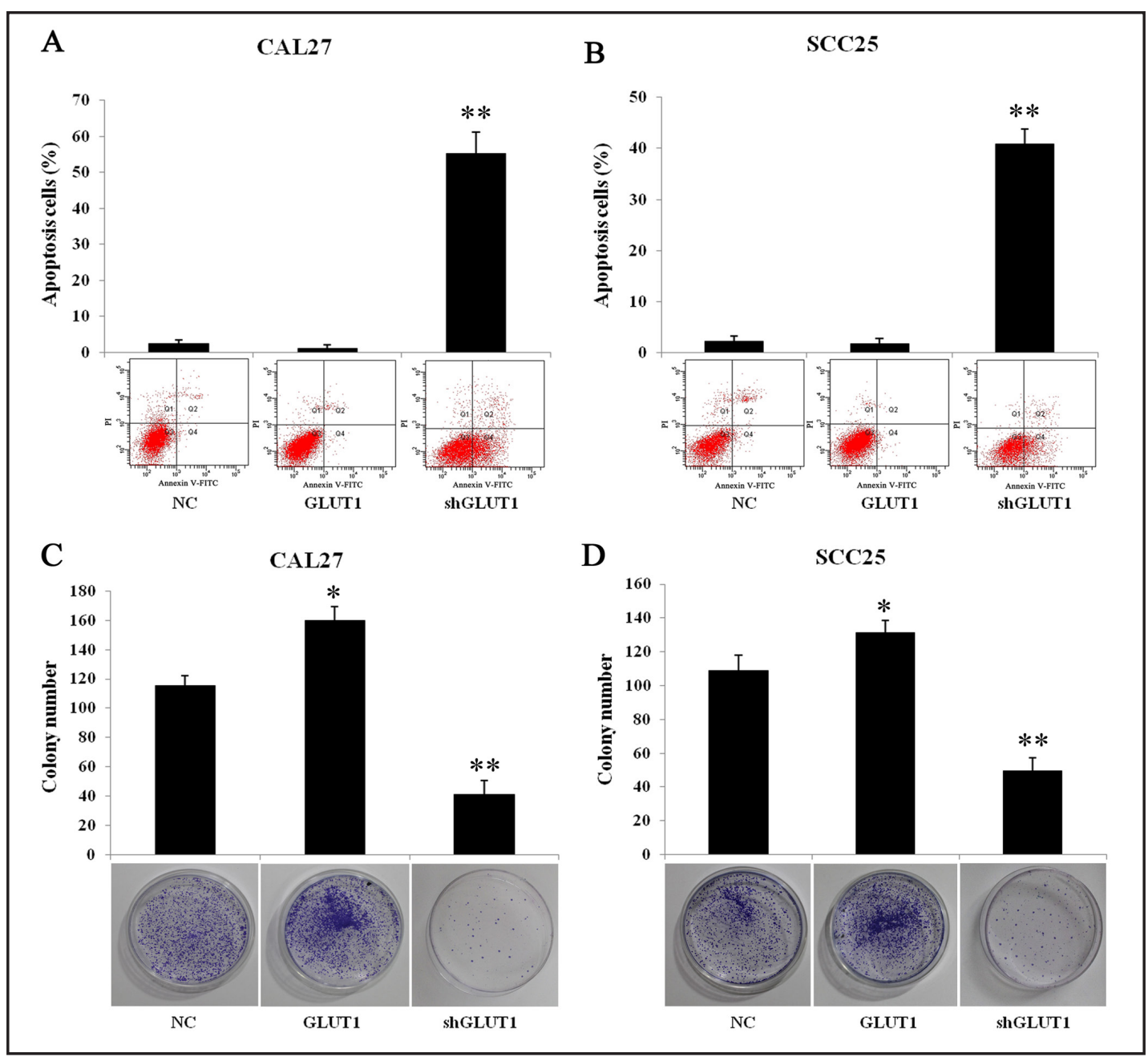

Fig. 2. GLUT1 expression is associated with apoptosis and colony formation ability in oral cancer cell lines. (A-B) Apoptosis was detected in CAL27 (A) and SCC25 (B) cells transfected as indicated by Annexin V-FITC staining and flow cytometry. Quantification of the percentage of Annexin V positive cells in each cell line subjected to different treatments. (C-D) Cells transfected as indicated and colony forming ability was assessed by staining with $0.5 \%$ crystal violet. Stained colonies larger than $1 \mathrm{~mm}$ in diameter of CAL27 (C) and SCC25 (D) cells were counted. All values are the means \pm SD of three replicates, ${ }^{*} \mathrm{P}<0.05,{ }^{* *} \mathrm{P}<0.01 \mathrm{VS}$ NC of each cell line.

lower in Lenti-shGLUT1 treated cells than in untreated cells in both cell lines (Fig. 3A). GLUT1 overexpression in CAL27 and SCC25 cells increased NFKB-p65 mRNA by 1.75- and 1.5fold $(\mathrm{P}<0.05)$, respectively, compared to the NC. These results were confirmed by Western blot analysis, which showed significant reductions in NFkB-p65 subunit protein levels in response to GLUT1 silencing $(\mathrm{P}<0.05)$, whereas GLUT1 overexpression caused a significantly increase in NFкB-p65 protein levels in both cell lines $(\mathrm{P}<0.05$, Fig. 3B). Taken together, these results indicate that the effects of GLUT1 on cell viability and apoptosis in HNSCC cells are associated with the modulation of NFKB-activity.

GLUT1 expression modulates tumorigenesis and survival in a mouse xenograft model of HNSCC

Xenograft tumors were generated in nude mice by injection of untransfected (NC) or different lenti-vector transfected CAL27 cells, and tumor growth and animal survival were monitored. At the end of the treatment period, mice were sacrificed and tumor volume was 
Fig. 3. The effects of GLUT1 expression are associated with the NFKB pathway. (A) Cells transfected as indicated were cultured for $72 \mathrm{~h}$ and NFKB-p65 mRNA levels were assessed by QRT-PCR, and normalized to GAPDH expression. mRNA levels are expressed as fold-change relative to the normal control group (NC). (B) NFKB-p65 protein levels were assessed by Western blotting. GAPDH was used as a loading control. Quantification of protein levels was performed by normalizing to the level of GAPDH. All values are the means \pm SD of three replicates, ${ }^{*} \mathrm{P}<0.05$ VS NC of each cell line.
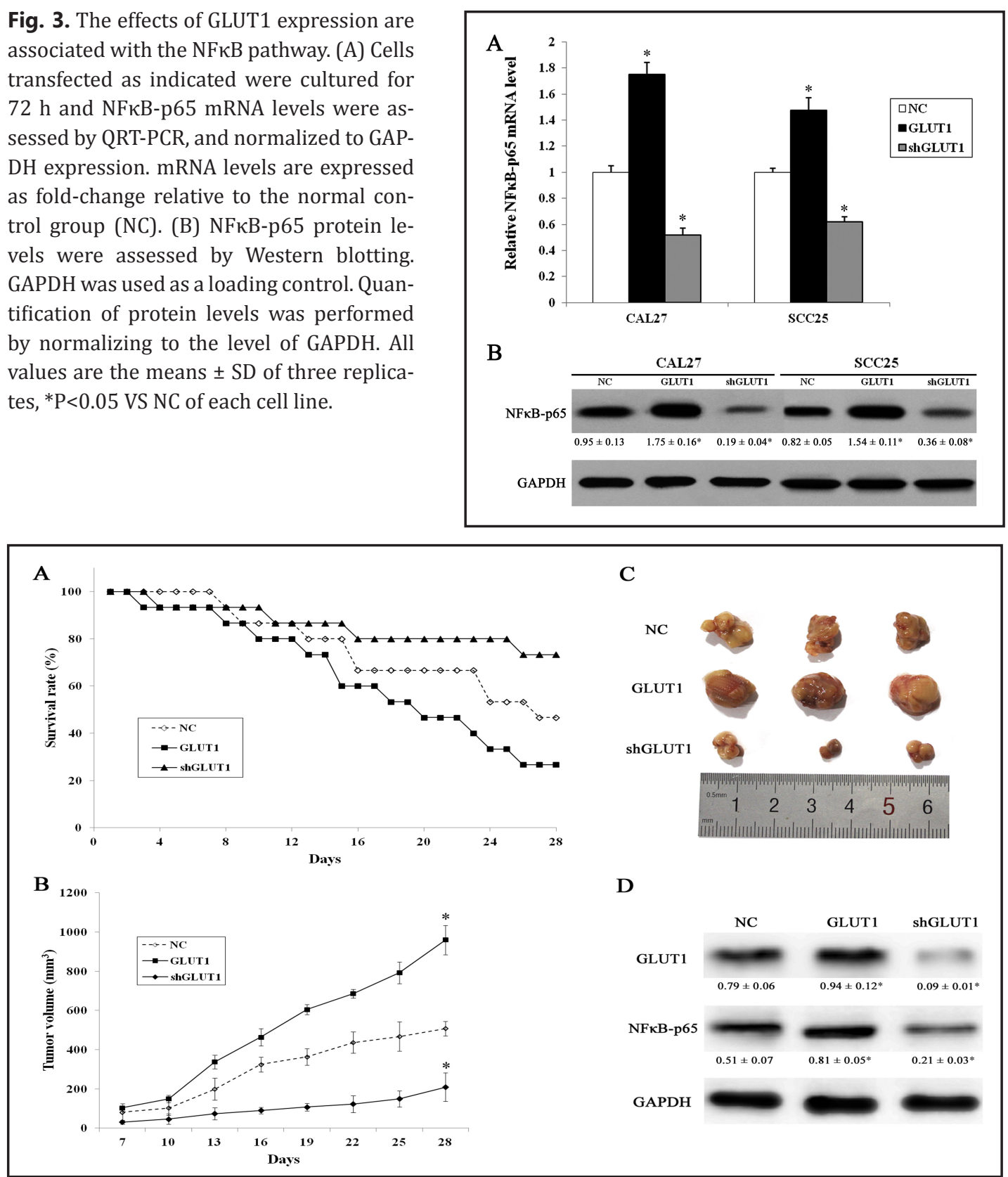

Fig. 4. GLUT1 expression is associated with tumor growth and survival in a xenograft mouse model. (A) Five week-old female athymic nude mice were injected subcutaneously as indicated to generate xenograft tumors and survival was monitored every day for 28 days. (B) One week after implantation, tumor sizes were measured with electronic digital calipers every three days for 21 days. (C) After 28 days post implantation, tumor xenografts were harvested. Representative image of tumors formed. (D) Snap-frozen tumors were analyzed by Western blotting against GLUT1 and NFKB-p65. GAPDH was used as a loading control. Quantification of protein levels was performed by normalizing to the expression of GAPDH. All values are the means \pm SD of three replicates, ${ }^{*} \mathrm{P}<0.05$ VS NC (implanted with untransfected CAL27 cells).

measured, and tumor tissues were analyzed by Western blotting. Fig. 4A shows the survival curves comparing mice bearing tumors. The results showed that the survival rate of xenograft mice was increased by GLUT1 silencing and reduced by GLUT1 overexpression. Assessment of changes in tumor volume over 21 days showed a significantly faster growth of Lenti- 
GLUT1 (GLUT1) xenograft tumors and an inhibition of growth in Lenti-shGLUT1 (shGLUT1) tumors compared to the NC tumors $(\mathrm{P}<0.05$, Fig. 4B). Fig. $4 \mathrm{C}$ shows representative tumors excised from the different xenograft mice. Tumor tissues were analyzed by Western blotting against GLUT1 and NFкB-p65, which showed a similar pattern to that observed in CAL27 cells subjected to the indicated treatments (Fig. 4D). GLUT1 overexpression significantly increased GLUT1 and NFKB-p65 levels $(\mathrm{P}<0.05)$, and GLUT1 silencing caused a significant reduction in GLUT1 expression and NFKB-p65 levels $(\mathrm{P}<0.05)$ compared to the NC group.

\section{Discussion}

In the early 1920s, Otto Warburg published the results of a study linking metabolism and cancer through an increase in the rate of aerobic glycolysis, which distinguishes cancer from normal tissues $[23,24]$. In what is known as the Warburg effect, reliance on aerobic glycolysis for the production of ATP is associated with the activation of oncogenes or inhibition of tumor suppressors [6]. Cancer cells acquire genetic mutations that alter signaling pathways and constitutively activate the uptake of nutrients, in particular glucose, to meet the increased bioenergetic demands of rapidly growing cells [5, 25]. In tumors, the increase in glucose uptake is achieved through the upregulation of facilitative glucose transporters, in particular GLUT1, which is overexpressed in a variety of malignancies and is a predictor of poor prognosis [26]. In the present study, we show that the effect of GLUT1 expression on resistance to chemotherapy and HNSCC cell viability and apoptosis occurs through a mechanism that involves NFKB activation.

We used two HNSCC cell lines to show that GLUT1 overexpression promotes cell viability whereas GLUT1 silencing decreases cell viability through the induction of apoptosis. In vivo experiments confirmed these results, showing increased survival and decreased xenograft tumor volume in association with GLUT1 silencing. Increased expression of GLUT1 has been detected in several types of cancer and overexpression of this transporter has been correlated with poor prognosis. In mouse mammary carcinoma, GLUT1 is the most abundantly expressed transporter and its silencing inhibits tumor growth whereas its overexpression promotes tumorigenesis in a mouse xenograft model [27]. In human breast carcinoma, GLUT1 expression is positively correlated with tumor grade and proliferative activity and is a marker of tumor aggressiveness and poor prognosis [7, 28]. GLUT1 mRNA levels are increased in hepatocellular carcinoma tissues and cell lines in association with a high proliferation index, advanced tumor stages and poor differentiation [29]. Haber et al. analyzed GLUT1 expression in 112 colorectal carcinoma samples in correlation with clinical outcome and showed that GLUT1 expression was associated with disease specific mortality, indicating that GLUT1 is a marker of poor prognosis [30]. High levels of GLUT1 expression were also detected in primary lung tumors and found to be a significant indicator of poor prognosis in non-small cell lung cancer [10, 31]. In head and neck cancer, Mellanen et al. suggested a relationship between GLUT1 expression and membrane localization and the uptake of glucose [9]. Data supporting a critical role of increased glucose transport and glucose metabolism in the progression of oral cancer were provided by several studies, in which GLUT1 expression was confirmed to be a negative biomarker of prognosis and survival $[11,12]$. Although the association of GLUT1 overexpression with tumorigenesis and progression of HNSCC has been described extensively, the mechanisms linking GLUT1 expression to tumor aggressiveness and poor survival remain unclear.

A number of mechanisms have been proposed to explain the regulation of GLUT1 expression in cancer. For example, hypoxia stabilizes HIF transcription factors resulting in the induction of GLUT1 expression [32]. A number of cancer genes that are associated with increased glucose uptake, including Myc, Akt, Ras and Raf among others, promote the localization of GLUT1 to the plasma membrane $[33,34]$ or induce transcription of the GLUT1 gene $[35,36]$. In the present study, we examined the mechanisms underlying the relationship of GLUT1 with resistance to cisplatin and the proliferation of HNSCC cells by focusing on the 
$\mathrm{NF \kappa B}$ pathway because of its known role in different aspects of cancer biology including angiogenesis, invasion, tumor proliferation and resistance to chemotherapy [37]. Our results showed that overexpression of GLUT1 significantly increased the levels of the NFkB-p65 subunit at the mRNA and protein levels whereas GLUT1 silencing had the opposite effect, indicating that an association between NFKB and GLUT1 exists in oral cancer. NFKB has been shown to control genes that negatively regulate apoptosis in tumor cells, thus promoting cancer cell survival [38]. Because of the crucial role of the NFKB pathway in the regulation of several cellular processes including apoptosis, its involvement in the induction of tumor cell proliferation by GLUT1 warrants further investigation.

Oncogenic transcription factors including $\mathrm{NF \kappa B}$ have been connected to several aspects of carcinogenesis, among them the resistance to chemotherapy [39]. NFKB was shown to induce drug resistance through inhibition of MDR1, which encodes the membrane transporter P-glycoprotein, in colon cancer cells [40] and inhibition of the NFKB pathway increases resistance to cisplatin in ovarian cancer [41]. Cisplatin is one of the most potent and commonly used antitumor agents in current clinical practice [42]. Therefore, the identification of cellular determinants of platinum compound sensitivity is critical to the design of therapeutic strategies in cancer. In the present study, we examined the potential association between GLUT1 expression and cisplatin resistance and showed that HNSCC cells with high levels of GLUT1 mRNA and protein expression showed significantly higher resistance to cisplatin compared to the corresponding parental lines. Similar results were reported by Shimanishi et al., who used RNAi mediated knock-down of GLUT1 and showed that inhibition of GLUT1 expression rendered oral cancer cells more sensitive to cisplatin under hypoxic conditions [43]. Further investigation of the association between GLUT1 expression and sensitivity to chemotherapeutic agents is important for the design of combination treatments in oral cancer.

In conclusion, silencing of GLUT1 expression in HNSCC cells by shRNA transfection decreased cell viability, reduced colony forming ability, and promoted apoptosis in CAL27 and SCC25 cells. In vivo experiments showed that knock-down of GLUT1 expression significantly decreased tumor volume and promoted survival in a mouse xenograft model. Overexpression of GLUT1, which promoted cancer cell viability and inhibited apoptosis, resulted in the activation of NFKB signaling, suggesting that the effect of GLUT1 in HNSCC may be associated with the NFkB pathway. Further investigation is necessary to understand the association between NFKB and GLUT1, which may help the design of therapeutic strategies targeting GLUT1 expression and improve the efficacy of chemotherapeutic drugs for the treatment of HNSCC.

\section{Acknowledgements}

This study was supported by grants from the National Natural Science Foundation of China (No. 81102047), the Fundamental Research Funds for the Central Universities, and the Shanghai Municipal Natural Science Foundation (No. 10ZR1432900).

\section{References}

Szablewski L: Expression of glucose transporters in cancers. Biochim Biophys Acta 2013;1835:164-169.

Pao SS, Paulsen IT, Saier MH Jr: Major facilitator superfamily. Microbiol Mol Biol Rev 1998;62:1-34.

Ganapathy V, Thangaraju M, Prasad PD: Nutrient transporters in cancer: Relevance to warburg hypothesis and beyond. Pharmacol Ther 2009;121:29-40.

Medina RA, Owen GI: Glucose transporters: Expression, regulation and cancer. Biol Res 2002;35:9-26.

Hsu PP, Sabatini DM: Cancer cell metabolism: Warburg and beyond. Cell 2008;134:703-707.

Vander Heiden MG, Cantley LC, Thompson CB: Understanding the warburg effect: The metabolic requirements of cell proliferation. Science 2009;324:1029-1033. 
Li/Yang/Wang/Ran: Relationship between GLUT1 and HNSCC

7 Kang SS, Chun YK, Hur MH, Lee HK, Kim YJ, Hong SR, Lee JH, Lee SG, Park YK: Clinical significance of glucose transporter 1 (glut1) expression in human breast carcinoma. Jpn J Cancer Res 2002;93:1123-1128. Haber RS, Weiser KR, Pritsker A, Reder I, Burstein DE: Glut1 glucose transporter expression in benign and malignant thyroid nodules. Thyroid 1997;7:363-367.

-9 Mellanen P, Minn H, Grenman R, Harkonen P: Expression of glucose transporters in head-and-neck tumors. Int J Cancer 1994;56:622-629.

10 Kurata T, Oguri T, Isobe T, Ishioka S, Yamakido M: Differential expression of facilitative glucose transporter (glut) genes in primary lung cancers and their liver metastases. Jpn J Cancer Res 1999;90:1238-1243.

11 Kunkel M, Reichert TE, Benz P, Lehr HA, Jeong JH, Wieand S, Bartenstein P, Wagner W, Whiteside TL: Overexpression of glut- 1 and increased glucose metabolism in tumors are associated with a poor prognosis in patients with oral squamous cell carcinoma. Cancer 2003;97:1015-1024.

12 Mineta H, Miura K, Takebayashi S, Misawa K, Araki K, Misawa Y, Ueda Y: Prognostic value of glucose transporter 1 expression in patients with hypopharyngeal carcinoma. Anticancer Res 2002;22:3489-3494.

13 Aggarwal S, Takada Y, Singh S, Myers JN, Aggarwal BB: Inhibition of growth and survival of human head and neck squamous cell carcinoma cells by curcumin via modulation of nuclear factor-kappab signaling. Int J Cancer 2004;111:679-692.

14 Jemal A, Siegel R, Xu J, Ward E: Cancer statistics, 2010. CA Cancer J Clin 2010;60:277-300.

15 Cmelak AJ: Current issues in combined modality therapy in locally advanced head and neck cancer. Crit Rev Oncol Hematol 2012;84:261-273.

16 Kim HJ, Hawke N, Baldwin AS: Nf-kappab and ikk as therapeutic targets in cancer. Cell Death Differ 2006;13:738-747.

17 Wang YD, Li SJ, Liao JX: Inhibition of glucose transporter 1 (glut1) chemosensitized head and neck cancer cells to cisplatin. Technol Cancer Res Treat 2013, epub ahead of print.

- 18 Fan J, Zhou JQ Yu GR, Lu DD: Glucose transporter protein 1-targeted rna interference inhibits growth and invasion of the osteosarcoma cell line mg63 in vitro. Cancer Biother Radiopharm 2010;25:521-527.

19 Kapoor V, Zaharieva MM, Das SN, Berger MR: Erufosine simultaneously induces apoptosis and autophagy by modulating the akt-mtor signaling pathway in oral squamous cell carcinoma. Cancer Lett 2012;319:3948.

20 Mosmann T: Rapid colorimetric assay for cellular growth and survival: Application to proliferation and cytotoxicity assays. J Immunol Methods 1983;65:55-63.

21 Wang K, Tang Y, Sun M, Lu B, Zhu H, Ji O, Shen Q: The mechanism of neogambogic acid-induced apoptosis in human mcf-7 cells. Acta Biochim Biophys Sin 2011;43:698-702.

22 Lee TK, Poon RT, Wo JY, Ma S, Guan XY, Myers JN, Altevogt P, Yuen AP: Lupeol suppresses cisplatin-induced nuclear factor-kappab activation in head and neck squamous cell carcinoma and inhibits local invasion and nodal metastasis in an orthotopic nude mouse model. Cancer Res 2007;67:8800-8809.

23 Dang CV: Links between metabolism and cancer. Genes Dev 2012;26:877-890.

24 Warburg 0: On respiratory impairment in cancer cells. Science 1956;124:269-270.

25 DeBerardinis RJ, Lum JJ, Hatzivassiliou G, Thompson CB: The biology of cancer: Metabolic reprogramming fuels cell growth and proliferation. Cell Metab 2008;7:11-20.

-26 Oliver RJ, Woodwards RT, Sloan P, Thakker NS, Stratford IJ, Airley RE: Prognostic value of facilitative glucose transporter glut-1 in oral squamous cell carcinomas treated by surgical resection; results of eortc translational research fund studies. Eur J Cancer 2004;40:503-507.

27 Young CD, Lewis AS, Rudolph MC, Ruehle MD, Jackman MR, Yun UJ, Ilkun O, Pereira R, Abel ED, Anderson SM: Modulation of glucose transporter 1 (glut1) expression levels alters mouse mammary tumor cell growth in vitro and in vivo. PLoS One 2011;6:e23205.

28 Younes M, Brown RW, Mody DR, Fernandez L, Laucirica R: Glut1 expression in human breast carcinoma: Correlation with known prognostic markers. Anticancer Res 1995;15:2895-2898.

29 Amann T, Maegdefrau U, Hartmann A, Agaimy A, Marienhagen J, Weiss TS, Stoeltzing O, Warnecke C, Scholmerich J, Oefner PJ, Kreutz M, Bosserhoff AK, Hellerbrand C: Glut1 expression is increased in hepatocellular carcinoma and promotes tumorigenesis. Am J Pathol 2009;174:1544-1552.

-30 Haber RS, Rathan A, Weiser KR, Pritsker A, Itzkowitz SH, Bodian C, Slater G, Weiss A, Burstein DE: Glut1 glucose transporter expression in colorectal carcinoma: A marker for poor prognosis. Cancer 1998;83:3440 . 
Li/Yang/Wang/Ran: Relationship between GLUT1 and HNSCC

-31 Younes M, Brown RW, Stephenson M, Gondo M, Cagle PT: Overexpression of glut1 and glut3 in stage I nonsmall cell lung carcinoma is associated with poor survival. Cancer 1997;80:1046-1051.

32 Semenza GL: Regulation of cancer cell metabolism by hypoxia-inducible factor 1. Semin Cancer Biol 2009;19:12-16.

-33 Rathmell JC, Fox CJ, Plas DR, Hammerman PS, Cinalli RM, Thompson CB: Akt-directed glucose metabolism can prevent bax conformation change and promote growth factor-independent survival. Mol Cell Biol 2003;23:7315-7328.

-34 Wieman HL, Wofford JA, Rathmell JC: Cytokine stimulation promotes glucose uptake via phosphatidylinositol-3 kinase/akt regulation of glut1 activity and trafficking. Mol Biol Cell 2007;18:14371446.

35 Yun J, Rago C, Cheong I, Pagliarini R, Angenendt P, Rajagopalan H, Schmidt K, Willson JK, Markowitz S, Zhou S, Diaz LA Jr, Velculescu VE, Lengauer C, Kinzler KW, Vogelstein B, Papadopoulos N: Glucose deprivation contributes to the development of kras pathway mutations in tumor cells. Science 2009;325:1555-1559.

- 36 Osthus RC, Shim H, Kim S, Li Q, Reddy R, Mukherjee M, Xu Y, Wonsey D, Lee LA, Dang CV: Deregulation of glucose transporter 1 and glycolytic gene expression by c-myc. J Biol Chem 2000;275:21797-21800.

37 Sethi G, Sung B, Aggarwal BB: Nuclear factor-kappab activation: From bench to bedside. Exp Biol Med (Maywood) 2008;233:21-31.

38 Aggarwal BB: Nuclear factor-kappab: The enemy within. Cancer Cell 2004;6:203-208.

39 Kohno K, Uchiumi T, Niina I, Wakasugi T, Igarashi T, Momii Y, Yoshida T, Matsuo K, Miyamoto N, Izumi H: Transcription factors and drug resistance. Eur J Cancer 2005;41:2577-2586.

-40 Bentires-Alj M, Barbu V, Fillet M, Chariot A, Relic B, Jacobs N, Gielen J, Merville MP, Bours V: Nf-kappab transcription factor induces drug resistance through mdr1 expression in cancer cells. Oncogene 2003;22:90-97.

-41 Mabuchi S, Ohmichi M, Nishio Y, Hayasaka T, Kimura A, Ohta T, Saito M, Kawagoe J, Takahashi K, YadaHashimoto N, Sakata M, Motoyama T, Kurachi H, Tasaka K, Murata Y: Inhibition of nfkappab increases the efficacy of cisplatin in in vitro and in vivo ovarian cancer models. J Biol Chem 2004;279:23477-23485.

42 Siddik ZH: Cisplatin: Mode of cytotoxic action and molecular basis of resistance. Oncogene 2003;22:72657279.

43 Shimanishi M, Ogi K, Sogabe Y, Kaneko T, Dehari H, Miyazaki A, Hiratsuka H: Silencing of glut-1 inhibits sensitization of oral cancer cells to cisplatin during hypoxia. J Oral Pathol Med 2013;42:382-388. 